\title{
Generational differences in workplace motivation
}

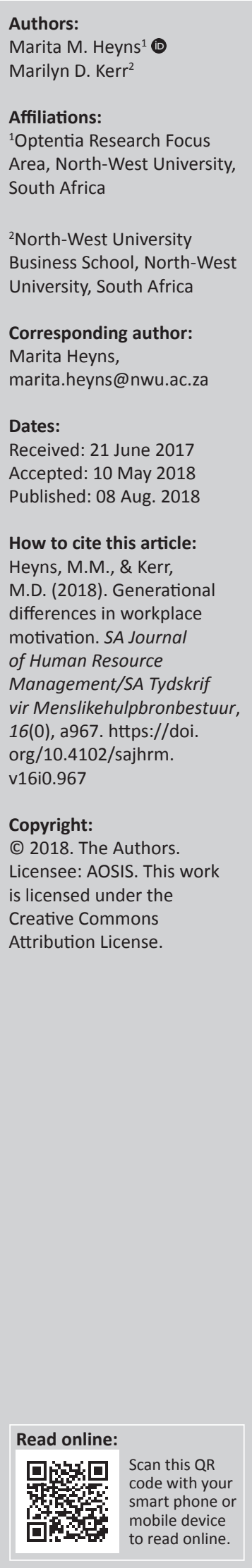

Orientation: Despite increasing age diversity in the workforce, organisations still know relatively little about how potentially diverging motivational needs of the various generations might influence motivational strategies and organisational performance.

Research purpose: To explore the relationship between multigenerational workforces and employee motivation within a South African workplace setting from a self-determination theory perspective.

Motivation for the study: The pursuit of performance excellence requires an understanding of the enablers of optimal performance. In South Africa, the workplace landscape is changing fast as younger generations are joining the workforce in rapidly growing numbers. These younger employees are often believed to differ quite drastically from the older generations in terms of their values and priorities, which necessitates a deeper understanding of the motivational drivers of the different cohorts as these manifest within a workplace environment.

Research approach/design and method: A cross-sectional survey approach and a quantitative research design were used $(N=164)$. Two questionnaires founded on self-determination theory were administered, namely the Work-Related Basic Need Satisfaction Scale and the Work Extrinsic and Intrinsic Motivation Scale.

Main findings: Findings contradict the popular notion that generational cohorts differ significantly from each other in terms of diverging intrinsic and extrinsic motivational preferences that may influence their behaviour at work. With regard to the degrees of satisfaction of the basic psychological needs that drive autonomous, intrinsically motivated behaviour specifically, no practically significant differences were found either. There was, however, one notable difference, namely in the indicated degree of satisfaction of the psychological need for autonomy between Generation Y and Generation X cohorts.

Practical/managerial implications: Management is advised to cultivate a motivational climate that promotes autonomously motivated behaviour in general and to focus on specific known individual motivational preferences that may exist within groups rather than approaching generational cohorts as homogenous groups.

Contribution/value-add: This study contributes to the limited research regarding similarities and differences in the intrinsic versus extrinsic motivational stance of three different generations as these manifest within a workplace setting in an emerging economy country. Findings afford management insight into motivational processes that are most influential among generational cohorts and assist them in adapting suitable motivational strategies that can ultimately improve retention of valued employees.

\section{Introduction}

\section{Key focus of the study}

This study seeks to extend generational research by employing a self-determination theory (SDT, Deci \& Ryan, 2000; Gagné \& Deci, 2005) perspective to examine the relationship between multigenerational workforces and employee motivation within a South African workplace setting.

\section{Background}

The pursuit of performance excellence requires an understanding of the enablers of optimal performances (Linley, Harrington \& Garcia, 2013), especially in view of a changing workplace landscape where increasing age diversity necessitates a deeper understanding of the needs and values of the different cohorts (Martins \& Martins, 2014).

Changing workforce demographics has become a particular concern for leadership because now, for the first time, there exists the possibility of four age generations working side by side in 
today's work environment (Ballone, 2007; Haynes, 2011). The increasing mix of generations has added both cherished diversity as well as complexity to the workplace (Linley et al., 2013) because of more pronounced differences between cohorts that influence their attitudes and behaviour at work as compared to previous generations, which were more similar to each other (Burke, Cooper \& Antoniou, 2015).

The different cohorts - Veterans, Baby Boomers, Generation Xers and Generation Y (also known as the Millennials) are often described as each having their own distinct characteristics, work values and motivators that may have an effect on both individual and organisational performance (Burke et al., 2015) and that may have far-reaching implications, including for organisational human resource processes (Jonck, Van der Walt \& Sobayeni, 2017), motivation and retention strategies (Burke et al., 2015). A longitudinal study by Krahn and Galambos (2014), for example, associated Generation Y with a stronger emphasis on extrinsic work values and more job entitlement, while other studies associated this generation with a greater preference for materialistic rewards and work-life balance (Burke et al., 2015). In line with this view, Twenge and Donnelly (2016) found that younger generations increasingly emphasise extrinsic values.

The perceived uniqueness of the generations suggests each is likely driven by different motivators, which, in turn, accentuates the need to have insight into how to best motivate each generational group (Durkin, 2011).

Organisations still know relatively little about the impact of age diversity on their performance and how to deal with generational diversity in the workplace (Burke et al., 2015; Martins \& Martins, 2014). Linley et al. (2013) illuminate this point by warning that, although some differences across generations do affect each cohort in distinctive ways, there are also indications that many of the perceived differences including those in values and attitudes towards work, colleagues and use of technology - across generations can actually be ascribed to stereotypical myths or, at best, to the influence of different contexts.

There is a specific need to conduct generational cohort studies in developing countries (Jonck et al., 2017; Martins \& Martins, 2014) and especially in a South African context, for it has faced some unique challenges because of its politically and socially divided past, which has caused fragmentation - not all social groups, including generations, have been affected by historical events in the same way. Also, most of the existing generational research studies have been conducted in developed Western countries, which raises concern regarding the generalisability of findings to developing countries such as South Africa (Jonck et al., 2017).

In addition, previous research focused on broad differences in motivational drivers across generations but paid scant attention to how these manifest within the workplace or, at best, tended to focus on work values but not on generational differences in work motivation per se (Burke et al., 2015; Wong, Gardiner, Lang \& Coulon, 2008).

This study focused on motivational levels of the multigenerational workforce of Rand Water, a parastatal and a national key point that is responsible for supplying quality water to millions of households in South Africa. Rand Water is invested in supporting the development of the younger generations and those previously disadvantaged in South Africa. Older employees also remained loyal to Rand Water, which has resulted in a work environment that sees employees representing a broad range of age groups. As Rand Water continued to focus on the empowerment of staff across generations, they found themselves in a situation where an abundance of resources was a thing of the past, and they were forced to work with less to create higher levels of output. This global trend aims towards increasing work output levels among employees, while facing challenges such as increased multigenerational workforces and the need to effectively motivate each generation, has created an urgent need for an understanding of how to motivate employees to the point that they bring $100 \%$ of themselves to their work.

Self-determination theory is a prominent, macro theory of motivation (Deci \& Ryan, 2014; Gagné \& Deci, 2005) that offers a potentially useful lens through which potential differences in the motivational stance across generations can be studied within the organisation of interest. This theory distinguishes itself from more conventional motivation theories because SDT not only expanded on the cognitive evaluation theory to include extrinsic motivation, but it further provides a distinction between autonomous and controlled motivation that does not focus on the total amount of motivation as such but rather on the relative strength of controlled versus autonomous motivation a person experiences. In other words, SDT does not merely focus on motivation as internally or externally driven but further differentiates between subtypes of motivation that are seen as falling along a continuum of internalisation (Deci \& Ryan, 2000; 2014; Gagné \& Deci, 2005). As such, SDT can offer more precise information regarding how generational groups relate to specific drivers of motivation.

\section{Research objectives}

The general aim of this research was to explore the nature of motivational processes from a multigenerational workforce perspective by using SDT (Deci \& Ryan, 2000) as a point of departure. To be more precise, this study assesses whether generations differ in terms of their extrinsic versus intrinsic motivational stance and their basic intrinsic motivational needs for autonomy, relatedness and competence as these manifest among the workforce of an organisation that supplies quality water to millions of households in South Africa.

\section{Trends from the research literature}

A generation, also known as a cohort, shares a collective identity that was brought about by shared life stages that 
shaped the culture of a particular historical period (Hoole \& Bonnema, 2015). Although there is no specific consensus among researchers regarding the precise birth years for each of the different generations (Wong et al., 2008), there is agreement that there are four broad generations of employees that can be classified, of which the three groups most represented in the workplace are the Baby Boomers (generally accepted to be born between 1945 and 1964), Generation Xers (born 1965-1981) and the Millennials (born 1982-2000).

A strong internalised work ethic and career-focused approach to life have meant a perception of the Baby Boomers as ambitious, driven employees who are status conscious (Ballone, 2007; Hoole \& Bonnema, 2015) and define their identity as an extension of their careers (Durkin, 2011). A review of the existing literature (e.g. Hart, 2006; Loomis, 2000;) suggests that this group of employees is seen as preferring stability and job security, that they respect a corporate hierarchal structure and prefer a leadership style that is unified and consistent in the work environment (Ballone, 2007). Baby Boomers gravitate towards building consensus among their colleagues (Hart, 2006:26). Valuing the personal touch (Haynes, 2011) and preferring face-to-face contact (Hammill, 2005) mean that this generation is more likely to act as effective mentors. It is through work and personal sacrifice (Glass, 2007) that Baby Boomers believe they will attain financial success. They are motivated by raises and promotions (Ballone, 2007). It is likely that Baby Boomers feel the younger generations do not work as hard as they do, partially derived from the latter group's preference for flexible hours, working from home and for having virtual offices (Glass, 2007).

In stark contrast with the Baby Boomers, Generation Xers value the life-work balance above all else (Glass, 2007; Hoole \& Bonnema, 2015). As a group, they are classified as showing a higher degree of scepticism, less loyalty and being strongly independent (Burke et al., 2015; Glass, 2007), as well as demonstrating a higher level of self-sufficiency than shown by previous generations (Hart, 2006). Although a tendency to question and challenge their colleagues in the workplace is apparent, thus potentially leading to conflict, this quality also acts to encourage entrepreneurial behaviour (Hammill, 2005). Generation Xers expect recognition and rewards to be realised within a short time frame, they expect to be included in all aspects of the business and to be provided with regular opportunities for career growth (Ballone, 2007). Placing their own personal goals above their work-related goals, this group goes where the challenges, higher earning potential and better benefits exist (Loomis, 2000). Flexibility in work life is greatly valued by Generation Xers and they are likely to pass up a promotion if they believe it will infringe on their home life in any way (Ballone, 2007). From their viewpoint, it does not matter how or where the work is done; the outcome is what should be valued and not the process to get there (Glass, 2007).

The most confident of the generational groups, the Millennials, grew up with child-focused parents, who were intent on building their children's self-esteem and showing continuous dedication in raising them (Glass, 2007). As a consequence, this generation is characterised by their expectation to be recognised on an equal footing with their peers and to be involved in a work environment that is diverse and encourages participation in work teams (Ballone, 2007; Hoole \& Bonnema, 2015). They have developed the ability to multitask, a consequence of $24 / 7$ connectivity (Hammill, 2005). Skills development and the challenges afforded by new opportunities typify Millennials (Hart, 2006). Demonstrating some similarity with the Baby Boomer generation, Millennials are seen as optimistic and driven employees who are demanding of their work environment (Burke et al., 2015). Ultimately, for this generation technology forms a natural part of their lives, and as such they prefer to communicate and live in real time, through the use of cell phones, text messages and so on (Ballone, 2007), rather than picking up the telephone or having a face-to-face conversation (Glass, 2007).

\section{Self-determination theory}

According to SDT, motivation can be internally or externally driven. Extrinsic motivation occurs when individuals partake in activities not because they have a particular interest in them but because those activities function as a means to an end. The actions undertaken by individuals driven by intrinsic motivation, on the other hand, are fuelled by the want to do the activity and the satisfaction derived from the successful completion of the task; thus, intrinsic motivation can be said to be autonomous motivation (Deci \& Ryan, 2014; Ryan \& Deci, 2000). The key to autonomous regulation is based on satisfaction of the basic psychological needs for competence, autonomy and relatedness. Autonomy is the need to feel that you have a choice in the decision to be made; competency is a belief in one's ability to complete a task, and relatedness is the need for relationships that are supportive and meaningful in nature (Deci \& Ryan, 2014; Meyer \& Gagné, 2008; Ryan \& Deci, 2000).

Autonomously motivated employees are likely to realise better outcomes for both themselves and their employers, through improved effective performance and higher levels of well-being (Gagné \& Deci, 2005; Nel, 2014). Limited satisfaction of the psychological needs is likely to result in a decreased level of performance and even a reduction in an individual's physical and psychological well-being (Meyer \& Gagné, 2008). Many organisational studies using the SDT have provided support for the hypothesised contention that environments that are autonomy-supportive facilitate the promotion of intrinsic motivation, as well as the internalisation of extrinsic motivation, thus acting to increase satisfaction and performance outcomes (Deci \& Ryan, 2014; Van den Broeck, Vansteenkiste, De Witte, Soenens \& Lens, 2010; Vansteenkiste et al., 2007).

Organismic Integration Theory is a sub-theory of SDT that aims to explain how behaviours once extrinsically motivated through external forces can become internally regulated via an internalisation process. Figure 1 illustrates the relationship 


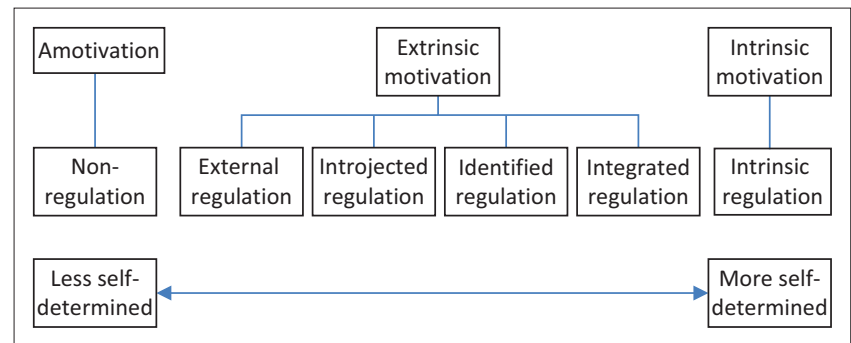

Source: Adapted from Ryan, R. M., \& Deci, E. L. (2000). Self-determination theory and the facilitation of intrinsic motivation, social development, and well-being. American Psychologist, 55(1), 68-78. https://doi.org/10.1037/0003-066X.55.1.68. p. 72.

FIGURE 1: The self-determination continuum.

between the various motivational aspects and describes the degree to which external regulation can be internalised in a self-determination continuum (Ryan \& Deci, 2000). It is not suggested the continuum is developmental in such a way that employees need to progressively move from controlled motivation to autonomously motivated behaviour through each stage of internalisation; rather that internalisation of a more autonomous behavioural regulation can occur at any point on the SDT continuum given specific individual life experiences and opportunities presented by the immediate environmental circumstances (Ryan \& Deci, 2000).

Amotivation and intrinsic motivation, being the two extremes of the continuum, respectively represent the total lack of intention to act (amotivation), that is, going through the motions, and a highly autonomous state (intrinsic motivation) characterised by the desire to perform a task purely for its inherent satisfactions (Ryan \& Deci, 2000).

In the centre of the continuum are the extrinsically motivated behaviours, which range from lowest self-determined behaviour to the highest - specifically, from external regulation through to integrated regulation. External regulation refers to behaviours that are controlled by external contingencies - the individual feels forced to comply. Each progressive rightward move along the continuum involves increased levels of personal acceptance and ownership of an external regulation, into the creation of an autonomous form of extrinsic motivation. Introjected regulation refers to behaviours that are still motivated by external rewards or punishments, but performance of the activities are controlled by the individual self rather than by others, that is, for an internally pressing reason. Identified regulation refers to a process that involves accepting the underlying value of an activity, such as when the goal of behaviour is personally endorsed and considered important, but extrinsic factors still play some role. Integrated regulation refers to the most complete form of internalisation of extrinsic motivation, namely, an acceptance of an activity because it fits into the individual's set of values and beliefs and is congruent with the true self (Nel, 2014; Ryan \& Deci, 2017).

Ultimately, the basic premise of SDT is the degree to which behaviour may be autonomous versus the degree to which it is controlled, where autonomous motivation includes intrinsic motivation as well as identified and integrated regulation and controlled motivation encompasses external and introjected regulation (Gagné \& Deci, 2005). Quoting Kasser et al. (2004), Vansteenkiste et al. (2007) state that individuals who are more likely to be intrinsically rather than extrinsically orientated will engage in those activities that function to satisfy their psychological needs, ultimately leading to positive job outcomes. For the converse, a mindset that is extrinsically orientated may hinder or interfere with growth (Vansteenkiste et al., 2007).

\section{Relationship between motivation and age groups}

No previous studies could be identified that examined potential generational differences specifically from an SDT motivational perspective. When considering motivation in general, previous research seems to produce conflicting results regarding the extent to which generations differ in what they consider as important motivational drivers. Kooij, De Lange, Jansen, Kanfer and Dikkers (2011) and De Lange, Bal, Van der Heiden, De Jong and Schaufeli (2011) have provided empirical evidence that supports the viewpoint that, while extrinsic work motivation decreases with age, intrinsic work motivation does the opposite and in fact increases. In examining demographic correlations that may exist among age groups and intrinsic motivation, a significant positive correlation was demonstrated between age and intrinsic motivation; however, no such parallel correlation was observed for age groups and extrinsic motivation.

Giancola (2006) provided an opposing viewpoint and stated that the differences perceived among the different generations were not substantiated by empirical evidence and in fact motivational drivers among the cohorts are actually surprisingly similar. Support for Giancola's viewpoint was provided by Wong et al. (2008), who, after conducting a large-scale cross-sectional study, were able to conclude that the differences identified among motivators were better explained by age differences rather than generational differences. Finegold, Mohrman and Spreitzer (2002) were able to identify significant differences among different age groups regarding a number of working relationships, including satisfaction with work-life balance, but found no significant relationship between generational groups and motivation. Brislin, Kabigting, MacNab, Zukis and Worthley (2005) and Travis (2007), in an examination of demographic correlations and intrinsic motivation among a sample population, found no significant correlation between the variables of age and motivation.

In view of seemingly conflicting results found in previous literature, this study sets out to investigate the following research questions: Do generational cohorts employed by Rand Water at the site of interest differ significantly from each other in terms of (1) their extrinsic versus intrinsic motivational stance and (2) the three basic intrinsic motivational needs for autonomy, relatedness and competence?

\section{Potential value-add of the study}

This research contributes to the existing generational literature by extending its focus to an emerging economy, specifically by 
highlighting similarities and differences in motivational stance between three generations - Baby Boomers, Generation Xers and Millennials - in a workplace setting that is considered to be of national interest to South Africa.

To the best of our knowledge, no previous empirical research exists that employed the SDT of motivation as proposed by Deci and Ryan (2000) to examine potential differences between generational groups within an emerging economy setting. Gaining a better and more specific understanding of what underlying motivational processes drive each generational cohort - whether it be an equal combination of intrinsic and extrinsic factors, or with one dominating the other - can support managers in finding suitable motivational strategies that are properly aligned with the needs of each cohort so that staff are more likely to function to their full potential and remain with an organisation in the longer term.

Findings may further afford management with insight into motivational processes that are most influential among generational cohorts at Rand Water, an important national asset of the country, and assist management in adapting suitable motivational strategies to enhance performance and ultimately improve retention of valued employees.

\section{Research design \\ Research approach}

A quantitative, cross-sectional survey design was used.

\section{Research participants}

The target population consisted of employees of a Rand Water pump station that is situated in Gauteng Province, South Africa. A total of 488 questionnaires were distributed, and 164 questionnaires were completed and returned, which represents a response rate of $33.6 \%$. More males (65\%) than females participated; $77 \%$ of the sample group indicated that they were black people. The majority $(48 \%)$ of the respondents indicated that they had a matric certificate as their highest qualification. When reviewing the duration of employment, $48 \%$ of the respondents indicated that they had been employed in excess of 10 years. Generation Xers represented the majority, with $59 \%$ of respondents falling within this category, followed by the Millennials (22\%) and the Baby Boomers (19\%).

\section{Measuring instruments}

A biographical questionnaire and two measuring instruments were used. The Work Extrinsic and Intrinsic Motivation Scale (WEIMS, Tremblay, Blanchard, Taylor, Pelletier \& Villeneuve, 2009) was employed to determine employees' motivational stance in terms of extrinsic versus intrinsic motivation. The WEIMS is an 18-item measure of work motivation theoretically grounded in SDT (Deci \& Ryan, 2000). The WEIMS is divided into six sub-scales (each with three corresponding items), corresponding to the six types of motivation postulated by SDT, namely, intrinsic motivation (e.g. 'because I derive much pleasure from learning new things'), integrated regulations (e.g. 'because it has become a fundamental part of who I am'), identified regulations (e.g. 'because this is the type of work I chose to do to attain a certain lifestyle'), introjected regulations (e.g. 'because I want to succeed at this job; if not I would be very ashamed of myself'), external regulations (e.g. 'for the income it provides me') and amotivation (e.g. 'I don't know; too much is expected of us'). The instrument makes use of a Likert-type scale ranging from 1 (does not correspond at all) to 5 (corresponds exactly), representing the reasons that the participants are presently involved in their work. Satisfactory alpha-coefficients with values $>0.7$ (Field, 2014) for each of the subsections were initially established by Tremblay et al. (2009).

In addition, the Work-Related Basic Need Satisfaction Scale (WBNSS; Van den Broeck et al., 2010) was used to measure each participant's satisfaction with three intrinsically motivating psychological needs, namely autonomy (e.g. 'I feel like I can be myself at my job'), competence (e.g. 'I am good at the things I do in my job') and need for relatedness (e.g. 'at work, people involve me in social activities'). The scale made use of a five-point rating scale, varying from 1 (totally disagree) to 5 (totally agree). CFA results favoured the three-factor structure of the questionnaire, and reliabilities of the autonomy, competence and relatedness satisfaction scales were on average $0.81,0.85$ and 0.82 , respectively (Van den Broeck et al., 2010).

\section{Research procedure}

Ethical clearance to conduct the study was approved by the School for Business and Governance of the North-West University of South Africa. The Human Resources director of the selected Rand Water plant provided permission for the questionnaire to be administered, in printed form, to the participants. The aggregated results of the study had to be provided to Rand Water for their own internal review. Participation was entirely voluntary and participants were aware that they could withdraw at any stage without penalty. After obtaining written consent, completion of the questionnaire took place during a predetermined time session following an introduction to the research content and purpose of the questionnaire by the researcher.

\section{Statistical analysis}

Two statistical programs - SPSS and AMOS - were employed. Descriptive statistics were used to describe the demographic data. Validity and reliability were tested by means of confirmatory factor analyses and the calculation of Cronbach's alpha values (Clark \& Watson, 1995) for all scales. Spearman's coefficient and analysis of variance (ANOVA) were used to analyse relationships between the sub-scales of the WBNSS and the WEIMS, respectively (Field, 2009). To interpret the practical significance of the findings, interpretation guidelines for Cohen's $d$-values (Ellis \& Steyn, 2003; Field, 2009) were followed, that is, $d \approx 0.2$ was regarded as a small effect (no practically significant difference), $d \approx 0.5$ as a medium effect (practically visible difference) and $d \approx 0.8$ as a large effect (practically significant difference). 


\section{Results}

\section{Descriptive statistics, internal consistencies and correlations}

Table 1 depicts the overall mean and standard deviation results per sub-scale for each of the two measurement instruments that were used to obtain an overall indication of the respondents' motivations for being involved in their work as postulated by SDT.

It is evident from the information provided on standard deviations in Table 1 that participants do not seem to differ drastically in their responses on the scales. The WEIMS results as shown in Table 1 implicate amotivation as the lowest ranking dimension with an average score of 3.28, implying the relative absence of motivation. In contrast, intrinsic motivation ranked higher than average. These results indicate that the respondents generally experienced a higher than average degree of intrinsic motivation towards their work. However, when reviewing the stages of the extrinsic motivation demonstrated on the SDT continuum, we find that introjected regulation ranks highest with a mean score of 5.43. This suggests that many employees are still in a process of adopting organisational rules but have not yet incorporated them into a sense of self - employees go along with the task because they believe they should and experience feelings of guilt if they do not.

The overall results for the WBNSS demonstrates that respondents are experiencing neutral feelings as far as the stimulation of intrinsic motivation is concerned. Competencea belief in one's ability to complete a task - with a mean value of 3.48 ranked the highest but also had the greatest standard deviation value, 1.02. The variation in the respondents' evaluation of the satisfaction of this need shows that although the majority of respondents feel neutral regarding competence, some employees feel less competent than the majority of employees.

Confirmatory factor analyses results supported the six-factor structure of the WEIMS as previously established by Tremblay et al. (2009) as well as the three-factor structure of the WBNSS as previously established by Van den Broeck et al. (2010).

TABLE 1: Descriptive statistics for employee motivation as measured by the Work Extrinsic and Intrinsic Motivation Scale and Work-Related Basic Need Satisfaction Scale.

\begin{tabular}{lcc}
\hline Dimensions & Mean & Standard deviation \\
\hline WEIMS & & \\
Intrinsic motivation & 5.17 & 0.15 \\
Integrated regulation & 5.07 & 0.36 \\
Identified regulation & 4.97 & 0.37 \\
Introjected regulation & 5.43 & 0.30 \\
External regulation & 4.82 & 0.40 \\
Amotivation & 3.28 & 0.53 \\
WBNSS & & \\
Autonomy & 3.33 & 0.33 \\
Competence & 3.48 & 1.02 \\
Need for relatedness & 2.91 & 0.70 \\
\hline
\end{tabular}

WEIMS, Work Extrinsic and Intrinsic Motivation Scale; WBNSS, Work-Related Basic Need Satisfaction Scale.
Considering the fact that psychological constructs were being measured and that Cronbach's alpha values for all scales were above or near the minimum required ( 0.7 as proposed by Field [2009]), the reliability of all the scales was considered acceptable.

Spearman's rho correlations were then analysed to determine whether there was a relationship between the drivers of intrinsic motivation as derived from the WBNSS and the motivation sub-scales as outlined by the WEIMS on the SDT continuum. As expected, the correlation coefficient $(r)$ analyses showed that intrinsic motivation had a medium (positive), practical and visible relationship with the three dimensions of autonomy, competence and relatedness. The results also showed medium, practically visible positive relationships across all three psychological needs for integrated regulation. The relationship observed between the drivers of intrinsically motivated behaviour and integrated regulation align with the literature, which states that integrated regulation is the stage on the SDT continuum that is closest to the fully autonomous intrinsic motivation.

Further examination showed that amotivation was inversely related to all three key psychological motivational needs. More specifically, amotivation had a medium (negative), practical and visible relationship with the psychological needs for autonomy and relatedness. In agreement with the SDT literature, external regulation demonstrated no practically significant relationship with the drivers of intrinsic motivation, as according to the continuum this stage is still predominantly a controlled behaviour.

\section{Basic psychological needs and generational groups}

ANOVA tests were used to compare the mean scores of the three generational cohorts in terms of intrinsic motivational drivers as measured by the WBNSS, extrinsic and intrinsic motivation at work and for the consideration of the subscales of extrinsic motivation measured in the WEIMS.

Table 2 gives an overview of differences among the cohorts for the entire motivational spectrum including intrinsic motivation, extrinsic motivation and amotivation as measured by the WEIMS. Although small effect size differences were noted for both intrinsic and extrinsic motivation between Generation $X$ and the Millennials, these were neither statistically significant nor would differences be observable between groups in practice.

Table 3 provides the results of each generation's answers for the sub-scales of extrinsic motivation as presented in the WEIMS questionnaire. These four sub-scales represent the internalisation of external regulation. The sub-scales move progressively from controlled (external regulation) to autonomous (integrated regulation) motivation. On average, introjected regulation was ranked the highest, suggesting that respondents take in a regulation but do not fully accept it as their own. 
TABLE 2: Descriptive statistics, analysis of variance and effect size results for extrinsic and intrinsic motivation.

\begin{tabular}{|c|c|c|c|c|c|c|c|}
\hline \multirow[t]{2}{*}{ Motivational type } & \multirow[t]{2}{*}{$N$} & \multirow[t]{2}{*}{ Mean } & \multirow[t]{2}{*}{ SD } & \multicolumn{2}{|c|}{ Effect sizes } & \multicolumn{2}{|c|}{ ANOVA } \\
\hline & & & & Millennials & Generation X & $F$ & Sig. \\
\hline \multicolumn{8}{|l|}{ Intrinsic motivation } \\
\hline Millennials & 36 & 5.18 & 1.17 & - & - & 0.83 & 0.44 \\
\hline Generation X & 94 & 5.00 & 1.19 & 0.25 & - & - & - \\
\hline Baby Boomers & 29 & 5.26 & 1.23 & 0.19 & 0.05 & - & - \\
\hline \multicolumn{8}{|l|}{ Extrinsic motivation } \\
\hline Millennials & 36 & 5.25 & 1.02 & - & - & 0.70 & 0.50 \\
\hline Generation X & 94 & 5.00 & 1.04 & 0.24 & - & - & - \\
\hline Baby Boomers & 29 & 5.09 & 1.13 & 0.14 & 0.07 & - & - \\
\hline \multicolumn{8}{|l|}{ Amotivation } \\
\hline Millennials & 36 & 3.17 & 1.48 & - & - & 0.21 & 0.75 \\
\hline Generation X & 94 & 3.26 & 1.44 & 0.06 & - & - & - \\
\hline Baby Boomers & 29 & 3.44 & 1.29 & 0.18 & 0.12 & - & - \\
\hline
\end{tabular}

$N$, number; $F$, indicates the test statistic of difference and is compared with the critical value of $F$ to determine its significance $p$; SD, standard deviation; sig., significance; ANOVA, analysis of variance test.

TABLE 3: Descriptive statistics, analyses of variances and effect size results for the sub-scales of extrinsic motivation.

\begin{tabular}{|c|c|c|c|c|c|c|c|}
\hline \multirow[t]{2}{*}{ Motivational type } & \multirow[t]{2}{*}{$N$} & \multirow[t]{2}{*}{ Mean } & \multirow[t]{2}{*}{ SD } & \multicolumn{2}{|c|}{ Effect sizes } & \multicolumn{2}{|c|}{ ANOVA } \\
\hline & & & & Millennials & Generation X & $\boldsymbol{F}$ & Sig. \\
\hline \multicolumn{8}{|c|}{ Integrated regulation } \\
\hline Millennials & 36 & 5.18 & 1.10 & - & - & 0.65 & 0.53 \\
\hline Generation X & 94 & 5.00 & 1.22 & 0.14 & - & - & - \\
\hline Baby Boomers & 29 & 5.26 & 1.12 & 0.07 & 0.21 & - & - \\
\hline \multicolumn{8}{|c|}{ Identified regulation } \\
\hline Millennials & 36 & 5.21 & 1.24 & - & - & 0.96 & 0.38 \\
\hline Generation X & 94 & 4.86 & 1.38 & 0.25 & - & - & - \\
\hline Baby Boomers & 29 & 5.06 & 1.31 & 0.11 & 0.14 & - & - \\
\hline \multicolumn{8}{|c|}{ Introjected regulation } \\
\hline Millennials & 36 & 5.66 & 1.06 & - & - & 1.03 & 0.36 \\
\hline Generation X & 94 & 5.38 & 1.21 & 0.23 & - & - & - \\
\hline Baby Boomers & 29 & 5.26 & 1.39 & 0.30 & 0.09 & - & - \\
\hline \multicolumn{8}{|l|}{ External regulation } \\
\hline Generation X & 94 & 4.77 & 1.27 & 0.14 & - & - & - \\
\hline Baby Boomers & 29 & 4.78 & 1.35 & 0.13 & 0.00 & - & - \\
\hline
\end{tabular}

$N$, number; $F$, indicates the test statistic of difference and is compared with the critical value of $F$ to determine its significance $p$; SD, standard deviation; sig., significance; ANOVA, analysis of variance test.

TABLE 4: Descriptive statistics and analysis of variance results for basic intrinsic motivational needs.

\begin{tabular}{|c|c|c|c|c|c|c|c|c|}
\hline \multirow[t]{2}{*}{ Dimension } & \multirow[t]{2}{*}{ Generation } & \multirow[t]{2}{*}{$N$} & \multirow[t]{2}{*}{ Mean } & \multirow[t]{2}{*}{ SD } & \multicolumn{2}{|c|}{ Effect sizes* } & \multicolumn{2}{|c|}{ ANOVA } \\
\hline & & & & & Millennials with & Generation $\mathrm{X}$ with & $F$ & Sig. \\
\hline \multirow[t]{3}{*}{ Autonomy } & Millennials & 36 & 3.47 & 0.69 & - & - & 1.64 & 0.20 \\
\hline & Generation X & 96 & 3.21 & 0.70 & 0.36 & - & - & - \\
\hline & Baby Boomers & 29 & 3.32 & 0.85 & 0.18 & 0.12 & - & - \\
\hline \multirow[t]{3}{*}{ Competence } & Millennials & 36 & 4.12 & 0.95 & - & - & 0.21 & 0.81 \\
\hline & Generation X & 94 & 4.11 & 0.77 & 0.00 & - & - & - \\
\hline & Baby Boomers & 29 & 4.22 & 0.68 & 0.11 & 0.14 & - & - \\
\hline \multirow{3}{*}{$\begin{array}{l}\text { Need for } \\
\text { relatedness }\end{array}$} & Millennials & 36 & 3.71 & 0.71 & - & - & 0.33 & 0.72 \\
\hline & Generation X & 95 & 3.60 & 0.73 & 0.15 & - & - & - \\
\hline & Baby Boomers & 29 & 3.64 & 0.64 & 0.10 & 0.06 & - & - \\
\hline
\end{tabular}

$N$, number; $F$, indicates the test statistic of difference and is compared with the critical value of $F$ to determine its significance $p$; SD, standard deviation; sig., significance; ANOVA, analysis of variance test.

WBNSS results for basic intrinsic motivational needs are displayed in Table 4.

As is evident from Table 4, there was no statistically significant difference in the way the different age groups responded to the relevant questions (all $p$-values $>0.05$ ) pertaining to the measurement of intrinsic motivational need satisfaction. The largest effect size difference $(d=0.36)$ was noted for the autonomy need, namely, between Millennials and Generation Xers. The difference highlights that Millennials express a higher satisfaction with the psychological need of autonomy than shown by generation Xers. In other words, Millennials felt that they had a higher sense of autonomy and that they had a choice in matters affecting them. All cohorts ranked the satisfaction of the psychological need for competence the highest, indicating that the respondents considered the ability to complete a task as highly important.

In sum, no statistically significant generational differences based on $p$-values and no medium or large differences indicated by the effect sizes were observed. Small effect size differences were noted between Millennials and Generation X 
on the Identified Regulation scale and between the Millennials and Generation X as well as Baby Boomers on the Introjected Regulation scale; however, none of these are of a practically significant magnitude. From these results, it appears that the generations are motivated similarly by intrinsic and extrinsic motivation. Overall, the Millennials presented higher average scores for both intrinsic and extrinsic motivation and the lowest means value for amotivation.

\section{Discussion}

The study tested whether there were generational differences among the workforce of a Rand Water plant in Gauteng in terms of their intrinsic and extrinsic motivation at work.

The proposed theoretical research model considered motivation in terms of autonomous and controlled motivation. For autonomous motivation, the degree of satisfaction of the psychological needs autonomy, competence and need for relatedness was considered. The stages of internalisation of extrinsic motivation were subdivided into autonomous and controlled forms of extrinsic motivation.

Prior to comparing cohorts, the relationship between the drivers of intrinsic motivational psychological needs and the motivation sub-scales on the SDT continuum was verified. As expected, the calculated correlation coefficients showed positive medium, practically visible relationships across all three psychological needs for integrated regulation. In further agreement with the SDT literature, external regulation demonstrated no practically significant relationship with the drivers of intrinsic motivation, as according to the continuum this stage is still predominantly a controlled behaviour.

From the literature, studies on the relationship between work motivation and age groups indicated two distinct strains of thought: firstly there are those who have identified differences and secondly those who believe the differences are negligible. This study found support for the latter of the two trains of thought, in that negligible differences between the groups were identified. In fact, a SDT perspective analyses showed that all three generational groups were motivated similarly by intrinsic and extrinsic motivation and all three groups indicated similar degrees of satisfaction with the three psychological needs related to autonomous, intrinsically motivated behaviour.

When contrasting specific cohorts in terms of their satisfaction of the basic psychological needs (autonomy, competence and relatedness), all groups indicated a strong belief in their ability to complete a task, as seen with the high ranking of the competency dimension. Similarly, all cohorts indicated a strong need for relatedness satisfaction. The only notable difference was found between the Millennials and Generation $X$ respondents in terms of the autonomy dimension. The Millennials indicated a higher sense of autonomy in their work when compared to the responses of the Generation $X$ cohort; however, the effect size indicated that the difference was still not of a practically visible magnitude. It is possible that because Generation Xers expect to be included in all aspects of the business (Ballone, 2007), which may prove difficult for the organisation to facilitate, the respondents may not feel like this need is being optimally fulfilled.

The fact that the generational cohorts experienced similar degrees of satisfaction of the basic intrinsically motivated psychological needs for autonomy, competence and relatedness provides further support for findings by Brislin et al. (2005) and Travis (2007), who found no significant correlation between the variables of age groups and motivation. Findings further suggest that the cohorts are influenced similarly by intrinsic as well as extrinsic motivation. This finding echoes the viewpoint set forward by Giancola (2006), where empirical evidence demonstrated that motivational drivers among generations were very similar. Findings also seem to resonate well with previous research by the Center for Creative Leadership, which found that younger and older generations seem to want similar things from their work and actually share many values in common (Linley et al., 2013).

Overall, the Millennials presented higher average scores for intrinsic and extrinsic motivation and the lowest mean value for amotivation. The aforementioned lends itself in support of the belief that intrinsic and extrinsic motivational rewards do not cancel out each other but work to maintain each other in a synergistic relationship (Khan \& Iqbal, 2013). The Millennials indicate on average the highest level of motivation, and the presented results show this is based on a relationship between intrinsic and extrinsic motivations. This conclusion indicates that in order to maintain the current motivation levels among the Millennials, the organisation needs to focus on both intrinsic and extrinsic rewards.

\section{Recommendations for the organisation}

Because findings concerning the organisation of interest revealed that younger and older generations actually are influenced similarly by extrinsic and extrinsic motivational drivers, managers are advised to refrain from relying on popular stereotypical ideas of generational differences when devising motivational strategies. According to SDT, motivational organisational contexts are best created by focusing on those psychological needs that form the basis for the direction and resolve for human behaviour - the needs for autonomy, relatedness and competence (Deci \& Ryan, 2000) - and satisfaction thereof will enhance both intrinsic motivation as well as the internalisation of extrinsic motivation (Gagné \& Deci, 2005). Enduring autonomous motivation can be promoted, for example, by creating psychologically safe and supportive yet challenging contexts that stimulate intrinsic interest, curiosity and creativity; by having meaningful discussions with employees regularly; allowing freedom of choice within a structure of clarified responsibilities; by providing a rationale for tasks and giving sincere feedback in a competent manner that is factual, nonjudgmental and free from demeaning criticism (Nel, 2014; Stone, Deci \& Ryan 2009). 
Because the observed trends in this study were not statistically significant, differences between individuals in terms of what motivates them may actually be of a greater magnitude within the same age group than those perceived between generations and warrants an exploration of the relative strength of specific motivational needs of individuals within a particular job context irrespective of age group. Discussions intended to motivate specific employees should seek to explore opportunities to satisfy intrinsic basic psychological needs according to the relative strength of those needs within the particular individual and as matched to the individual's specific work context. This implies that the organisation will have to invest more into understanding individuals' perspectives. According to Stone, Deci and Ryan (2009), meaningful discussions with employees are characterised by asking open questions, listening attentively, acknowledging employee perspectives and by refraining from pressure, threats and directives to perform.

It should nevertheless be noted that workplaces will continue to become even more multigenerational in the future (Haynes, 2011) and it is likely that stereotypes among age groups themselves will persist and may cause heightened sensitivity to perceived age discrimination (Burke et al., 2015). It is therefore recommended that the organisation should look for ways to foster a generational-friendly environment (Hoole \& Bonnema, 2015), such as to increase interaction among employees of different ages through inter-group activities, mentorship programmes and other initiatives. According to Next Step Growth (2013), the top suggestions for bridging generational gaps within the work environment include collaborative work styles and tools, team-building events, the use of the latest technology, leadership coaching and mentorship programmes.

\section{Study limitations and recommendations for future research}

The relatively small sample size and the sampling procedure used in the study did not ensure proportionate representation within each cohort and limits generalisations to the larger population. For future studies, the use of a stratified randomsample design may be beneficial in that it could ensure sufficient representation of the different generational groups in the larger selected population.

It is also acknowledged that a cross-sectional study cannot decisively establish whether observations of small differences that have been noted in some instances are a result of generation or age. Although a once-off measurement is useful as a starting point, it is necessary to explore the trends over time through longitudinal follow-up studies.

Another limitation of the study is that it applied age categorisations presented by the literature that tend to be used across the globe, yet this universal typology of generations may prove to be too simplistic. Because Burke et al. (2015, p. 156) emphasised that 'generational attributes by their very nature are specific to the socio-political events that create and shape these cohorts', more rigorous scientific verification is necessary to determine whether the broad age cut-off points used to differentiate cohorts from each other are indeed justifiable and applicable to the South African context. If potentially more appropriate cut-off points for age could be verified and used in future studies, it may for instance transpire that the current statistically non-significant yet consistent differences observed between Generation $Y$ and both Generation X and the Baby Boomers in terms of the relative strength of their motivational needs may reveal itself more clearly.

\section{Acknowledgements Competing interests}

The authors declare that they have no financial or personal relationships that may have inappropriately influenced them in writing this article.

\section{Authors' contributions}

M.H. was the project leader and is the corresponding author. M.K. prepared the initial analyses of results. Both authors made substantial conceptual contributions to this article.

\section{References}

Ballone, C. (2007). Consulting your clients to leverage the multi-generational workforce. Journal of Practical Consulting, 2(1), 9-15.

Brislin, R. W., Kabigting (Jr), F., MacNab, B., Zukis, B., \& Worthley, R. (2005). Evolving perceptions of Japanese workplace motivation: An employee-manager comparison. International Journal of Cross Cultural Management, 5(1), 87-104. https://doi.org/ $10.1177 / 1470595805050829$

Burke, R. J., Cooper, C. L., \& Antoniou, A. G. (2015). The multi-generational and aging workforce: Challenges and opportunities. Cheltenham, UK: Edward Elgar Publishing Ltd.

Clark, L.A. \& Watson, D. 1995. Constructing Validity: Basic Issues in Objective Scale Development. Psychological Assessment, 7(3): 309-319

Deci, E. L., \& Ryan, R. M. (2000). The 'what' and 'why' of goal pursuits: Human needs and the self-determination of behavior. Psychological Inquiry, 11, 227-268. https://doi.org/10.1207/S15327965PLI1104_01

Deci, E. L., \& Ryan, R. M. (2014). The importance of universal psychological needs for understanding motivation in the workplace. In M. Gagné (Ed.), The Oxford handbook of work engagement, motivation, and self-determination theory (pp. 13-32). New York, NY: Oxford University Press.

De Lange, A. H., Bal, P. M., Van der Heiden, B. I. J. M., De Jong, N., \& Schaufeli, W. B. (2011). When I'm 64: Psychological contract breach, work motivation and the moderating roles of future time perspective and regulatory focus. Work \& Stress, 25(4), 338-354. https://doi.org/10.1080/02678373.2011.632610

Durkin, D. (2011). Effectively managing the multigenerational workforce. Retrieved from $\mathrm{http}$ ///under30ceocom/effectively-managing-the-multigenerational-workforce.html

Ellis, S. M., \& Steyn, H. S. (2003). Practical significance (effect sizes) versus or in combination with statistical significance (p-values). Management Dynamics, 12(4), 51-53.

Field, A. 2014. Discovering statistics using IBM SPSS Statistics. 4th ed. London: UK. Sage

Finegold, D., Mohrman, S., \& Spreitzer, G. M. (2002). Age effects on the predictors of technical workers' commitment and willingness to turnover. Journal of Organizational Behavior, 23, 655-674. https://doi.org/10.1002/job.159

Gagné, M., \& Deci, E. L. (2005). Self-determination theory and work motivation. Journal of Organizational Behavior, 26(4), 331-362. https://doi.org/10.1002/job.322

Giancola, F. (2006). The generation gap: More myth than reality? Human Resource Planning, 29(4), 32-37.

Glass, A. (2007). Understanding generational differences for competitive success. Industrial and Commercial Training, 39(2), 98-103. https://doi.org/10.1108/ 00197850710732424

Hammill, G. (2005). Mixing and managing four generations of employees. Retrieved from http://www.fdu.edu/newspubs/magazine/05ws/generations.htm

Hart, K. A. (2006). Generations in the workplace: Finding common ground. Medical Laboratory Observer, 38(10), 26-27.

Haynes, B. P. (2011). The impact of generational differences on the workplace. Journal of Corporate Real Estate, 13(2), 98-108. https://doi.org/10.1108/1463001111 1136812 
Hoole, C., \& Bonnema, J. (2015). Work engagement and meaningful work across generational cohorts. SA Journal of Human Resource Management, 13(1). Art. generational cohorts. SA Journal of Human Resource
$\# 681,1-11$. https://doi.org/10.4102/sajhrm.v13i1.681

Jonck, P., Van der Walt, F., \& Sobayeni, N. C. (2017). A generational perspective on work values in a South African sample. SA Journal of Industrial Psychology, 43, a1393. https://doi.org/10.4102/sajip.v43.1393

Khan, W., \& Iqbal, Y. 2013. An investigation of the relationship between work motivation (intrinsic \& extrinsic) and employee engagement. Umea University: Umea School of Business (Thesis - MA)

Kasser, T., Ryan, R. M., Couchman, C. E., \& Sheldon, K. M. (2004). Materialistic values: Their causes and consequences. In T. Kasser, \& A. D. Kanner (Eds.), Psychology and consumer culture: The struggle for a good life in a materialistic world (pp. 11-28). Washington, DC: American Psychological Association.

Kooij, D. T. A. M., De Lange, A. H., Jansen, P. G. W., Kanfer, R., \& Dikkers, J. S. E. (2011). Age and work-related motives: Results of a meat-analysis. Journal of Organizational Behavior, 32(2), 197-225. https://doi.org/10.1002/job.665

Krahn, H. J., \& Galambos, N. L. (2014). Work values and beliefs of 'Generation X' and 'Generation Y'. Journal of Youth Studies 17(1), 92-112. https://doi.org/10.1080/1 3676261.2013.815701

Linley, P. A., Harrington, S., \& Garcea, N. (2013). The Oxford handbook of psychology and work. New York: Oxford University Press.

Loomis, J.E. (2000). Gen X, Rough Notes Co., Indianapolis, IN.

Martins, N., \& Martins, E. C. (2014). Perceptions of age generations regarding employee satisfaction in a South African organisation. Mediterranean Journal of Social Sciences, 5(21), 129-140. https://doi.org/10.5901/mjss.2014.v5n $21 \mathrm{p} 129$

Meyer, J. P., \& Gagné, M. (2008). Employee engagement from a self-determination theory perspective. Industrial and Organizational Psychology, 1, 60-62. https:// doi.org/10.1111/j.1754-9434.2007.00010.x

Nel, L. (2014). Theories on character strengths, hope and self-determination In M. Wissing, J. Potgieter, T. Guse, T. Khumalo, \& L. Nel (Eds.), Towards flourishing: Contextualising positive psychology, (pp. 115-140). Pretoria: Van Schaik Publishers.
Next Step Growth. (2013). The multi-generational workforce challenge. Retrieved from http://www.nextstepgrowth.com/wp-content/uploads/2013/01/Research Summary09.pdf

Ryan, R. M., \& Deci, E. L. (2000). Self-determination theory and the facilitation of intrinsic motivation, social development, and well-being. American Psychologist, 55(1), 68-78. https://doi.org/10.1037/0003-066X.55.1.68

Ryan, R. M., \& Deci, E. L. (2017). Self-determination theory: Basic psychological needs in motivation, development, and wellness. New York: The Guilford Press.

Stone, D. N., Deci, E. L., \& Ryan, R. M. (2009). Beyond talk: Creating autonomous motivation through self-determination theory. Journal of General Management 34, 75-91. https://doi.org/10.1177/030630700903400305

Travis, D. J. (2007). Is doing good enough? A path analytic model of intrinsic job satisfaction among human service workers. Journal of Workplace Behaviora Health, 22(1), 13-32. https://doi.org/10.1300/J490v22n01_02

Tremblay, M. A., Blanchard, C. M., Taylor. S., Pelletier, L. G., \& Villeneuve, M. (2009). Work extrinsic and intrinsic motivation scale: Its value for organisational psychology research. Canadian Journal of Behavioural Science, 41(4), 213-226. https://doi. org/10.1037/a0015167

Twenge, J. M \& Donnelly, K. (2016). Generational differences in American students' reasons for going to college, 1971-2014: The rise of extrinsic motives. The Journa of Social Psychology, 156(6), 620-629. https://doi.org/10.1080/00224545.2016.1 152214

Van den Broeck, A., Vansteenkiste, M., De Witte, H., Soenens, B., \& Lens. W. (2010) Capturing autonomy, competence, and relatedness at work: Construction and initial validation of the work-related basic need satisfaction scale. Journal of Occupational and Organizational Psychology, 83, 981-1002. https://doi.org/ $10.1348 / 096317909 \times 481382$

Vansteenkiste, M., Neyrinck, B., Niemiec, C. P., Soenens, B., De Witte, H., \& Van de Broeck, A. (2007). On the relations among work value orientations, psychological need satisfaction and job outcomes: A self-determination theory approach Journal of Occupational and Organizational Psychology, 80, 251-277. https://doi. org/10.1348/096317906X111024

Wong, M., Gardiner, E., Lang, W., \& Coulon, L. (2008). Generational differences in personality and motivation. Journal of Managerial Psychology, 23(8), 878-890. https://doi.org/10.1108/02683940810904376 